\title{
Telomere-to-telomere genome assembly of asparaginase-producing Trichoderma simmonsii
}

\author{
Dawoon Chung, Yong Min Kwon and Youngik Yang
}

\begin{abstract}
Background: Trichoderma is a genus of fungi in the family Hypocreaceae and includes species known to produce enzymes with commercial use. They are largely found in soil and terrestrial plants. Recently, Trichoderma simmonsii isolated from decaying bark and decorticated wood was newly identified in the Harzianum clade of Trichoderma. Due to a wide range of applications in agriculture and other industries, genomes of at least 12 Trichoderma spp. have been studied. Moreover, antifungal and enzymatic activities have been extensively characterized in Trichoderma spp. However, the genomic information and bioactivities of T. simmonsii from a particular marinederived isolate remain largely unknown. While we screened for asparaginase-producing fungi, we observed that $T$. simmonsii GH-Sj1 strain isolated from edible kelp produced asparaginase. In this study, we report a draft genome of T. simmonsii GH-Sj1 using Illumina and Oxford Nanopore technologies. Furthermore, to facilitate biotechnological applications of this species, RNA-sequencing was performed to elucidate the transcriptional profile of $T$. simmonsii GH-Sj1 in response to asparaginase-rich conditions.

Results: We generated $\sim 14 \mathrm{~Gb}$ of sequencing data assembled in a $\sim 40 \mathrm{Mb}$ genome. The T. simmonsii GH-Sj1 genome consisted of seven telomere-to-telomere scaffolds with no sequencing gaps, where the N50 length was 6.4 Mb. The total number of protein-coding genes was 13,120, constituting 99\% of the genome. The genome harbored 176 tRNAs, which encode a full set of 20 amino acids. In addition, it had an rRNA repeat region consisting of seven repeats of the 18S-ITS1-5.8S-ITS2-26S cluster. The T. simmonsii genome also harbored 7 putative asparaginase-encoding genes with potential medical applications. Using RNA-sequencing analysis, we found that 3 genes among the 7 putative genes were significantly upregulated under asparaginase-rich conditions.
\end{abstract}

Conclusions: The genome and transcriptome of T. simmonsii GH-Sj1 established in the current work represent valuable resources for future comparative studies on fungal genomes and asparaginase production.

Keywords: Trichoderma simmonsii, Telomere-to-telomere assembly, Comparative genome analysis, Asparaginase

\footnotetext{
*Correspondence: yiy@mabik.re.kr

National Marine Biodiversity Institute of Korea, Chungchungnam-do, South

Korea
}

(c) The Author(s). 2021 Open Access This article is licensed under a Creative Commons Attribution 4.0 International License, which permits use, sharing, adaptation, distribution and reproduction in any medium or format, as long as you give appropriate credit to the original author(s) and the source, provide a link to the Creative Commons licence, and indicate if changes were made. The images or other third party material in this article are included in the article's Creative Commons licence, unless indicated otherwise in a credit line to the material. If material is not included in the article's Creative Commons licence and your intended use is not permitted by statutory regulation or exceeds the permitted use, you will need to obtain permission directly from the copyright holder. To view a copy of this licence, visit http://creativecommons.org/licenses/by/4.0/ The Creative Commons Public Domain Dedication waiver (http://creativecommons.org/publicdomain/zero/1.0/) applies to the data made available in this article, unless otherwise stated in a credit line to the data. 


\section{Background}

Fungal species belonging to the genus Trichoderma produce a variety of valuable factors with different function, including enzymes [1]. For example, T. reesei is a representative cellulolytic microorganism used for the degradation of lignocellulosic plant materials. Cellulases from T. reesei and $T$. viride as well as chitinase, xylanase, and lysine oxidase from $T$. viride are already commercially available [2, 3].

In addition to enzymatic activities, various Trichoderma fungi have been extensively studied for their mycoparasitic properties. In particular, T. harzianum is used as a commercial biocontrol agent against plant diseases [4]. In fungal phylogeny, the Harzianum clade consists of at least 18 Trichoderma species, including $T$. harzianum, T. guizhouense, T. inhamatum, T. lentiforme, T. lixii, T. afarasin, T. afroharzianum, T. atrobrunneum, T. camerunense, T. endophyticum, T. neotropicale, T. pyramidale, T. rifaii, T. simmonsii [5], T. lentinulae, $T$. vermifimicola, T. xixiacum, and T. zelobreve [6]. Fungi in this clade are ubiquitous and often isolated from the soil as well as plant debris and occasionally from marine resources such as sediments and sea sponges [7, 8].

T. simmonsii is a newly described fungal species belonging to the Harzianum clade, mostly isolated from decaying bark and decorticated wood [5]. Since its first identification in the United States in 2015 (MycoBank MB809947), T. simmonsii strains have been reported in several countries in Europe and, more recently, in China and South Korea [9, 10]. This fungus was also identified in formulated biocontrol agents [5]. Furthermore, T. simmonsii strain UTFC 10063 efficiently accumulates cadmium in its biomass, exhibiting potential as a bioremoval agent in cadmium-polluted solutions [11]. However, when compared to other Trichoderma species, the molecular characteristics and bioactivities of T. simmonsii are poorly understood.

Fungal genome analyses have highlighted the genetic diversity within the fungal kingdom in addition to differences in fungal morphology, physiology, and ecology [12]. Due to advances in high-throughput sequencing technologies, the body of available fungal genome data is rapidly increasing. Recently, genomes of the most common 12 Trichoderma spp. including T. reesei, T. parareesei, T. longibrachiatum, T. citrinovirde, T. harzianum, T. afroharzianum, T. guizhouense, T. virens, T. asperellum, T. hamatum, T. atroviride, and T. gamsii, were compared in order to understand the evolution, core genome, and gene inventory of Trichoderma [13].

Our laboratory has screened different marine-derived fungi with enzymatic activities. L-asparaginase (L-asparagine amidohydrolase, EC 3.5.1.1) is an enzyme that hydrolyzes L-asparagine to aspartic acid and is utilized for the treatment of acute lymphoblastic leukemia [14] as well as for the reduction of carcinogenic compound acrylamide in food [15]. Currently, asparaginases from Escherichia coli and Erwinia chrysanthemi are utilized as therapeutic agents [16]. However, the discovery of novel asparaginases is necessary as bacterial asparaginase occasionally causes adverse effects, including allergic responses. In this study, we report the genomic analysis of marine-derived T. simmonsii isolate GH-Sj1, one of the fungal strains we screened for asparaginase activity. In addition, we performed transcriptomic analysis of GH-Sj1 under asparaginase-rich conditions. Although Trichoderma species are well-known valuable resources for industrial enzymes, their asparaginase production remains unexplored. Through this paper, we provide insights into the $T$. simmonsii genome as well as its expression profile under asparaginase-rich conditions.

\section{Results}

Identification of marine-derived T. simmonsii GH-Sj1

A marine-derived strain, designated $\mathrm{GH}-\mathrm{Sj} 1$, was isolated from a sea algae Saccharina japonica collected in Sacheon, Republic of Korea. This strain produced abundant aerial mycelia and whitish and green granular colonies on PDA at $25^{\circ} \mathrm{C}$ for 7 days (Fig. 1A). It produced subglobose to ovoid conidia in a green disk around the inoculum with sizes in the range of $2.5-3.0 \mu \mathrm{m}$ in width $\times 2.8-3.5 \mu \mathrm{m}$ in length $(N=10)$ (Fig. $1 \mathrm{~B})$. Conidiophores developed to form branches having a terminal whorl of multiple phialides (Fig. 1C). These morphological features of GH-Sj1 were similar to those of Trichoderma spp. previously reported [5].

Molecular identification was performed using a translation elongation factor $1 \alpha$ gene $(t e f 1 \alpha)$ sequence that is a widely used genetic marker for Trichoderma identification $[17,18]$. From the BLASTN search, tef1 $\alpha$ of GH-Sj1 showed a high degree of sequence identity to that of the T. simmonsii type specimen G.J.S. 91-138 (98.8\%, GenBank AF443935). In addition, GH-Sj1 tef1 $\alpha$ sequence was also similar to that of $T$. lentinulae CGMCC 3.19847 (94.8\%, GenBank MN605878), T. camerunense GJS 99-230 (94.0\%, GenBank AF348107), and T. harzianum CBS 226.95 (92.8\%, AF348101). Consequently, based on the morphological and molecular features, GH-Sj1 was identified as T. simmonsii.

\section{DNA sequencing of $T$. simmonsii isolate GH-Sj1}

To reconstruct the high quality genome of $T$. simmonsii GH-Sj1, we generated sufficient sequencing depth of Illumina short reads for high base accuracy [19] and Nanopore long reads to overcome fragmented assembly $[20,21]$. As a result, we obtained, in total, 7,521,311,812 bp from 24,905,006 PE reads using Illumina Technologies' short read sequencing platform, which resulted in a genome coverage of $\sim 187 x$. After trimming low-quality 
A

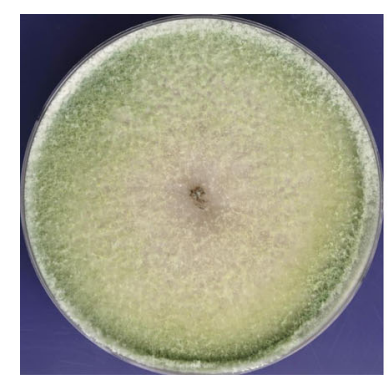

B

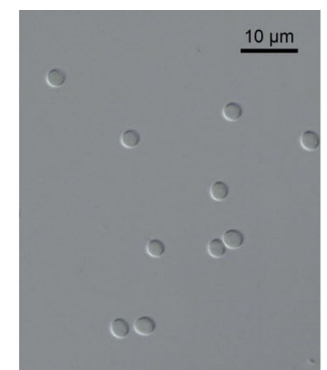

C

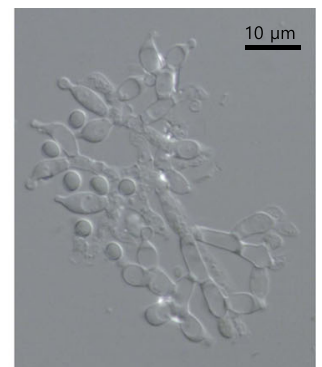

Fig. 1 Characterization of Trichoderma simmonsii GH-Sj1 morphology. (A) Colony of GH-Sj1 cultured on PDA at $25^{\circ} \mathrm{C}$ for 7 days. (B) Microscopic images of conidia. GH-Sj1 produces subglobose to ovoid conidia. (C) A conidiophore image of GH-Sj1

bases, 6,985,160,902 bp from 23,255,700 PE reads were retained, corresponding to $\sim 174 \mathrm{x}$ genome coverage. Utilizing the Oxford Nanopore Technologies' long read sequencing platform, 795,128 long reads $(7,510,994,507$ bp) were generated, with a genome coverage of $\sim 187 \mathrm{x}$. Finally, 795,176 reads $(7,480,287,989$ bp) remained after adapter trimming, covering $\sim 186 \mathrm{x}$ of the genome length.

\section{Genome assembly of $T$. simmonsii isolate GH-Sj1}

Since short length reads often leads to fragmented de novo assembly [21], we only used 239,681 Nanopore reads $(5,783,154,314 \mathrm{bp})$ with at least $10 \mathrm{~Kb}$ for the genome assembly which constituted a genome coverage of $\sim 144 \mathrm{x}$. We then compared the performances of multiple de novo assemblers in order to find the best draft assembly, which included Canu [22], Flye [23], Miniasm [24], Shasta [25], and Wtdbg2 (v2.3) [26]. These draft assemblies were polished using Nanopore long reads and Illumina short reads as described in the Methods section.

Supplementary Table 1 shows the results of the five draft assemblies. The assembly lengths, GC contents and BUSCO scores were comparable among the assemblers, where the values were approximately $40 \mathrm{Mb}, 48$, and $99 \%$, respectively. However, Miniasm output was best by several criteria. It generated the smallest number of contigs of 9 , of which 7 contigs were longer than $100 \mathrm{~Kb}$. L50 was the best with Flye and Wtdbg2 at 3. Moreover, five contigs were assembled telomere-to-telomere. We therefore chose Miniasm contigs as the primary assembly and refined the results as follows. Two overlapping contigs were merged as one scaffold. Conversely, a contig was splitted which assembled to two contigs in other assemblers. In addition, we dropped a very short contig and a mitochondrial sequence. For more details, refer to the Methods section. As a result, the final genome assembly consisted of seven genomic scaffolds (40,078,385 bp) with an N50 length of $6.4 \mathrm{Mb}$. The $T$. simmonsii genome contained no gaps, and the GC content was $48.13 \%$ as summarized in Table 1 . The average base coverage of genomes for Illumina WGS reads and Nanopore WGS reads were $\sim 168 \mathrm{x}$ and $\sim 186 \mathrm{x}$, respectively. There were a couple of regions where read coverages were exceptionally high. One of these was the rRNA repeat region in scaffold 5, where the maximum base coverage of Illumina and Nanopore reads was 4704x and 9668x, respectively. All seven scaffolds were in chromosome scale, wherein each scaffold is assembled telomere-to-telomere. Table 1 shows the occurrences of telomere repeats for each scaffold. The minimum telomere repeats were 12 , and the maximum were 17 . For the full alignments of telomere regions, refer to Supplementary Table 2. As pointed out in [27], telomere regions are highly AT-rich, illustrated in Fig. 2 as the fourth ring from outside to inside. Centromere regions are also very high in AT and scarce in genes. In terms of centromere positions, scaffold 1,2 , and 6 appeared to be metacentric where $\mathrm{p}$ and $\mathrm{q}$ arms are of compatible length, while the others were submetacentric, with the $\mathrm{p}$ arm being shorter than the $\mathrm{q}$ arm. For assembly completeness, our genome assemblies recovered $99 \%$ of the BUSCO [28] with regard to fungi_odb10 gene groups.

\section{Genome annotation of $T$. simmonsii isolate GH-Sj1}

After achieving the high quality assembly of T. simmonsii $\mathrm{GH}-\mathrm{Sj} 1$, we annotated the genome using funannotate pipeline which specialized for annotating fungal genomes [29]. As summarized in Table 2, 13,120 proteincoding genes $(13,875$ proteins) were predicted based on ab initio prediction and RNA-seq data. The number of

Table 1 Telomere repeat occurrences of Trichoderma simmonsii GH-Sj1

\begin{tabular}{llllllll}
\hline Terminus & Scaffold $\mathbf{1}$ & Scaffold $\mathbf{2}$ & Scaffold $\mathbf{3}$ & Scaffold $\mathbf{4}$ & Scaffold $\mathbf{5}$ & Scaffold $\mathbf{6}$ & Scaffold $\mathbf{7}$ \\
\hline $3^{\prime} \rightarrow 5^{\prime}(\mathrm{CCCTAA}) n$ & 13 & 12 & 14 & 12 & 12 & 17 & 13 \\
$5^{\prime} \rightarrow 3^{\prime}($ TTAGGG) $n$ & 13 & 14 & 15 & 15 & 12 & 15 & 16 \\
\hline
\end{tabular}




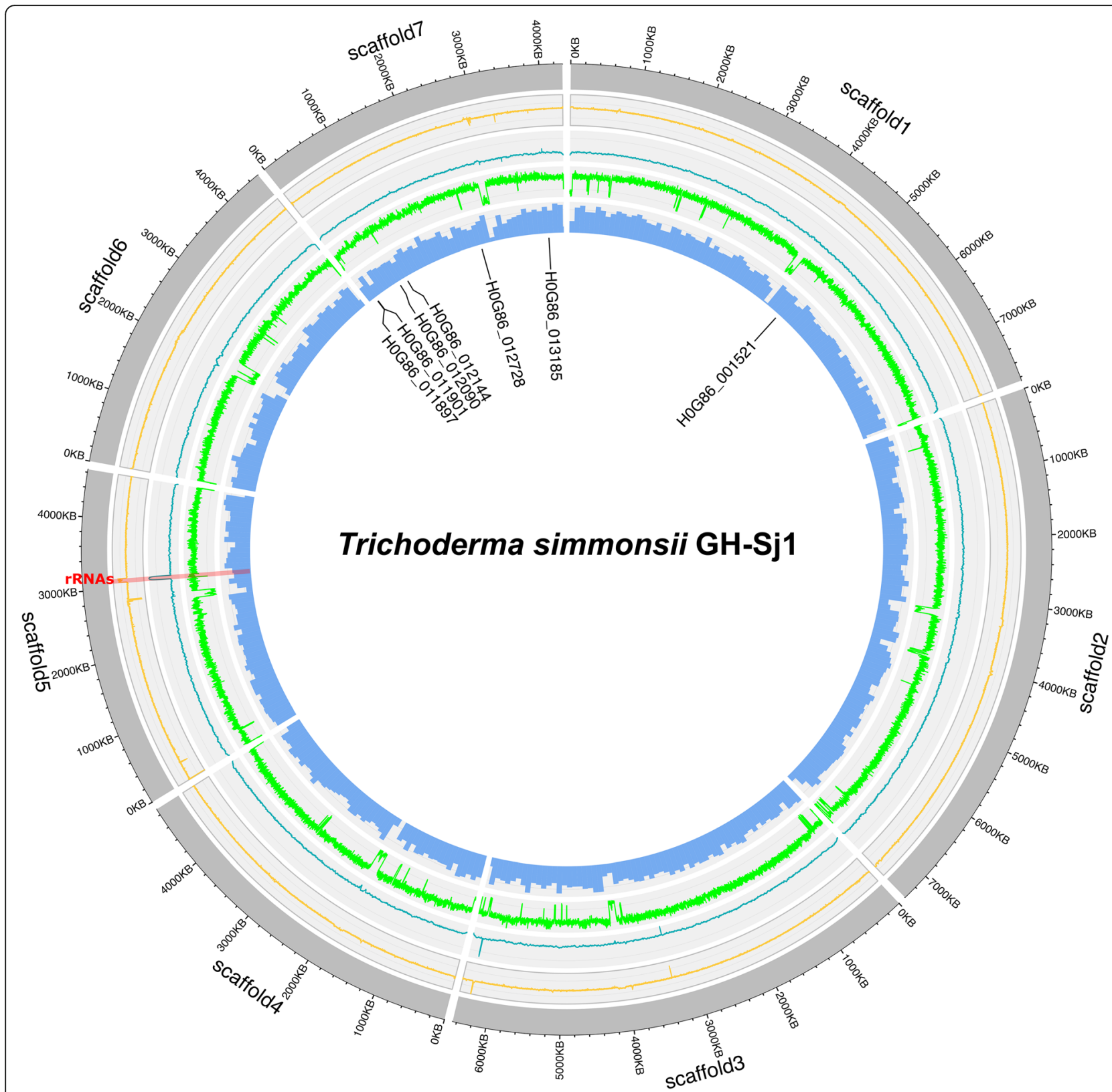

Fig. 2 Circular representation of the Trichoderma simmonsii GH-Sj1 genome. From outer ring to inner ring: first ring, scaffolds; second ring, log2 of Illumina WGS read coverages in $1 \mathrm{~Kb}$ windows; third ring, log2 of Nanopore WGS read coverages in $1 \mathrm{~Kb}$ windows; fourth ring, GC contents in 1 $\mathrm{Kb}$ windows; fifth ring, gene counts in $100 \mathrm{~Kb}$ windows. The tracks and labels of genes above the title: the locus of asparaginases

predicted genes of $T$. simmonsii were similar as for other Trichoderma species (Table 3). The average gene length was $1452 \mathrm{bp}$, average exon length was $645 \mathrm{bp}$, and the average number of exons per gene was 2.96 (Table 2). The T. simmonsii genome consisted of 176 tRNA genes where the full set of 20 amino acids were found along with three defined as tRNA-iMet (Supplementary Table 3). Scaffold 5 contained an rRNA repeat region, highlighted in Fig. 2, where 21 rRNAs were predicted. The region harbored seven repeats of rRNA clusters, with each repeat consisting of an 18S-ITS1-5.8S-ITS226S rRNA gene cluster. Clusters of Orthologous Groups of proteins (COG) [30] functional category distribution is shown in Table 4 and Supplementary Fig. 1. Disregarding (S) Function unknown, the top 5 functional categories were (Q) Secondary metabolites biosynthesis, transport and catabolism, (G) Carbohydrate transport and metabolism, $(\mathrm{O})$ Posttranslational modification, protein turnover, chaperones, (E) Amino acid transport and metabolism, and $(\mathrm{U})$ Intracellular trafficking, secretion, 
Table 2 Genome statistics of Trichoderma simmonsii GH-Sj1

\begin{tabular}{ll}
\hline Number of genomic scaffolds & $\mathbf{7}$ \\
\hline Total length (bp) & $40,078,385$ \\
N50 (bp) & $6,451,197$ \\
Number of N's (bp) & 0 \\
GC content (\%) & 48.13 \\
BUSCO (genome) & $98.7 \%$ \\
tRNA genes & 176 \\
rRNA genes & 21 \\
Number of protein coding genes & 13,120 \\
Number of proteins & 13,875 \\
Average gene length (bp) & 1452.7 \\
Average exon length (bp) & 645 \\
Average number of exons per gene & 2.96 \\
\hline
\end{tabular}

and vesicular transport. T. simmonsii was most annotated in all the COG categories except for (V) Defense mechanisms, where it was second only to $T$. harzianum. Carbohydrate-Active Enzymes (CAZyme) [31] classification results are presented to Supplementary Fig. 2 and 3. The occurrences of CAZyme categories Auxiliary Activities (AA), CarbohydrateBinding Modules (CBM), Carbohydrate Esterases (CE), Glycoside Hydrolases (GH), Glycosyl Transferases (GT), and Polysaccharide Lyases (PL) were 126, 12, 56, 423,78 , and 13, respectively. The top 10 most occurring families were CE10 (31), GH18 (24), AA7 (23), AA3 (20), GH3 (19), AA3_3 (19), AA3_2 (19), GH16 (16), GH2 (13), and GH55 (12), where the value in parenthesis represents the occurrence of each family. The antiSMASH results [32] are summarized in Supplementary Table 4, where the two most abundant cluster types were Type 1 Polyketide synthase (T1PKS) and Non-ribosomal peptide synthetase cluster (NRPS).

\section{Reference genomes}

To analyze the genomic similarities and differences between $T$. simmonsii $\mathrm{GH}-\mathrm{Sj} 1$ and related genomes, we collected 11 previously annotated Trichoderma genomes from NCBI: T. asperellum CBS 433.97, T. atroviride IMI 206040, T. citrinoviride TUCIM 6016, T. gamsii T6085, T. guizhouense NJAU 4742, T. harzianum CBS 226.95, T. lentiforme CFAM-422, T. longibrachiatum ATCC 18648, T. parareesei CBS 125925, T. reesei QM6a, and T. virens Gv29-8. Table 3 shows assembly statistics for T. simmonsii and the other 11 species. Compared to $T$. simmonsii GH-Sj1 ( $40 \mathrm{Mb})$, assembly lengths of the listed genomes ranged from $\sim 32 \mathrm{Mb}(T$. parareesei) to $\sim 41 \mathrm{Mb}$ ( $T$. harzianum). GC contents ranged from $47 \%$ to $\sim 53 \%$, and the T. simmonsii GC content ( 48\%) belongs to this range. The number of genes ranges from $\sim 9 \mathrm{~K}$ to $\sim 13 \mathrm{~K}$, where $T$. simmonsii had the second highest number of genes and proteins following $T$. harzianum.

\section{Phylogeny of $T$. simmonsii}

After investigating structure and compositions of the reference genomes, we questioned the evolutionary relationships of $T$. simmonsii with other Trichoderma spp. To answer the question, we constructed a maximum likelihood phylogenetic tree of $T$. simmonsii using RAxML [33] shown in Fig. 3 using the 11 reference Trichoderma species and F. oxysporum NRRL-32932 as an out-group. The divergence times of species were calculated via MEGA [34] using the estimated time between $T$. harzianum and $F$. oxysporum (98-269 million years ago (MYA)). The tree topology was concordant with that from a previous study [13]. T. simmonsii formed monophyletic groups with the Harzianum clade genomes of $T$. guizhouense, T. lentiforme, and T. harzianum along with the Virens clade genome of $T$. virens.

Table 3 Trichoderma genomes statistics

\begin{tabular}{|c|c|c|c|c|c|c|c|c|}
\hline Clade & Species & Strain & NCBI accession & $\begin{array}{l}\text { Genome } \\
\text { size }(\mathrm{Mb})\end{array}$ & Sca-ffold & GC (\%) & Genes & Proteins \\
\hline \multirow[t]{5}{*}{ Harzianum/Nirens } & T. simmonsii & GH-Sj1 & $\mathrm{N} / \mathrm{A}$ & 40.07 & 7 & 48.13 & 13,296 & 13,875 \\
\hline & T. guizhouense & NJAU 4742 & GCA_002022785.1 & 38.32 & 63 & 49.56 & 11,255 & 11,255 \\
\hline & T. harzianum & CBS 226.95 & GCF_003025095.1 & 40.98 & 532 & 47.58 & 14,269 & 14,065 \\
\hline & T. lentiforme & CFAM-422 & GCA_011066345.1 & 38.31 & 47 & 49.65 & 12,978 & 12,978 \\
\hline & T. virens & GV29-8 & GCF_000170995.1 & 39.02 & 93 & 49.25 & 12,405 & 12,406 \\
\hline \multirow[t]{4}{*}{ Longibrachiatum } & T. citrinoviride & TUCIM 6016 & GCF_003025115.1 & 33.21 & 533 & 52.31 & 9929 & 9735 \\
\hline & T. longibrachiatum & ATCC 18648 & GCA_003025155.1 & 32.23 & 130 & 51.25 & 11,132 & 10,934 \\
\hline & T. parareesei & CBS 125925 & GCA_001050175.1 & 32.07 & 885 & 53.47 & 9062 & 9062 \\
\hline & T. reesei & QM6a & GCF_000167675.1 & 33.39 & 77 & 52.75 & 9109 & 9111 \\
\hline \multirow[t]{3}{*}{ Trichoderma } & T. asperellum & CBS 433.97 & GCF_003025105.1 & 37.46 & 419 & 47.31 & 12,775 & 12,557 \\
\hline & T. atroviride & IMI 206040 & GCF_000171015.1 & 36.14 & 29 & 49.75 & 11,809 & 11,816 \\
\hline & T. gamsii & T6085 & GCF_001481775.2 & 37.90 & 172 & 48.95 & 11,171 & 11,171 \\
\hline
\end{tabular}




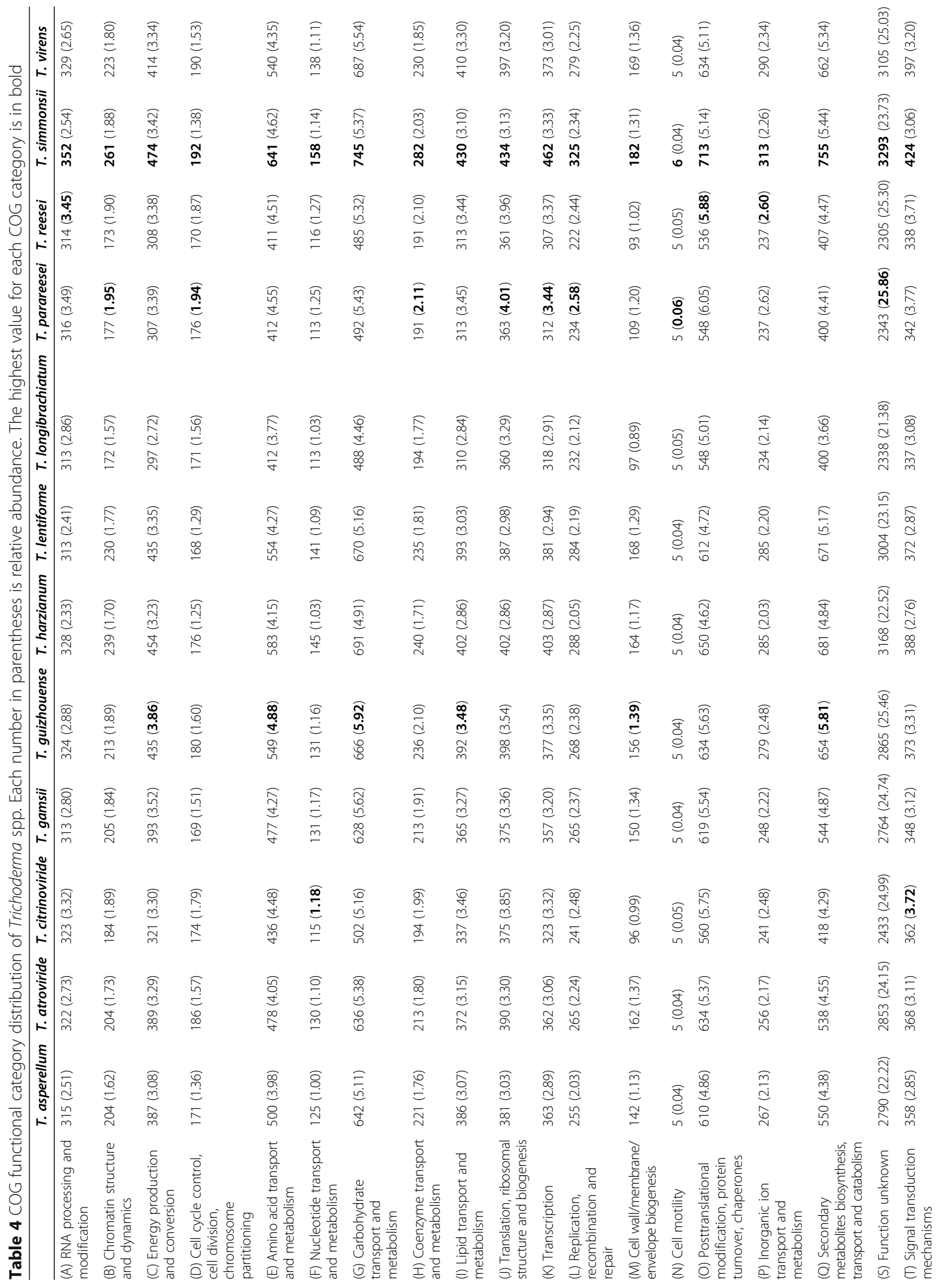




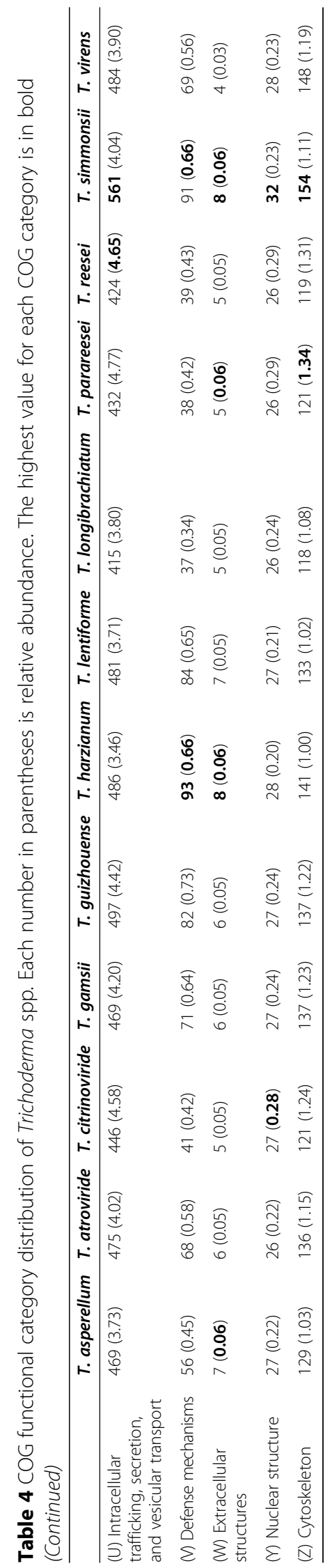




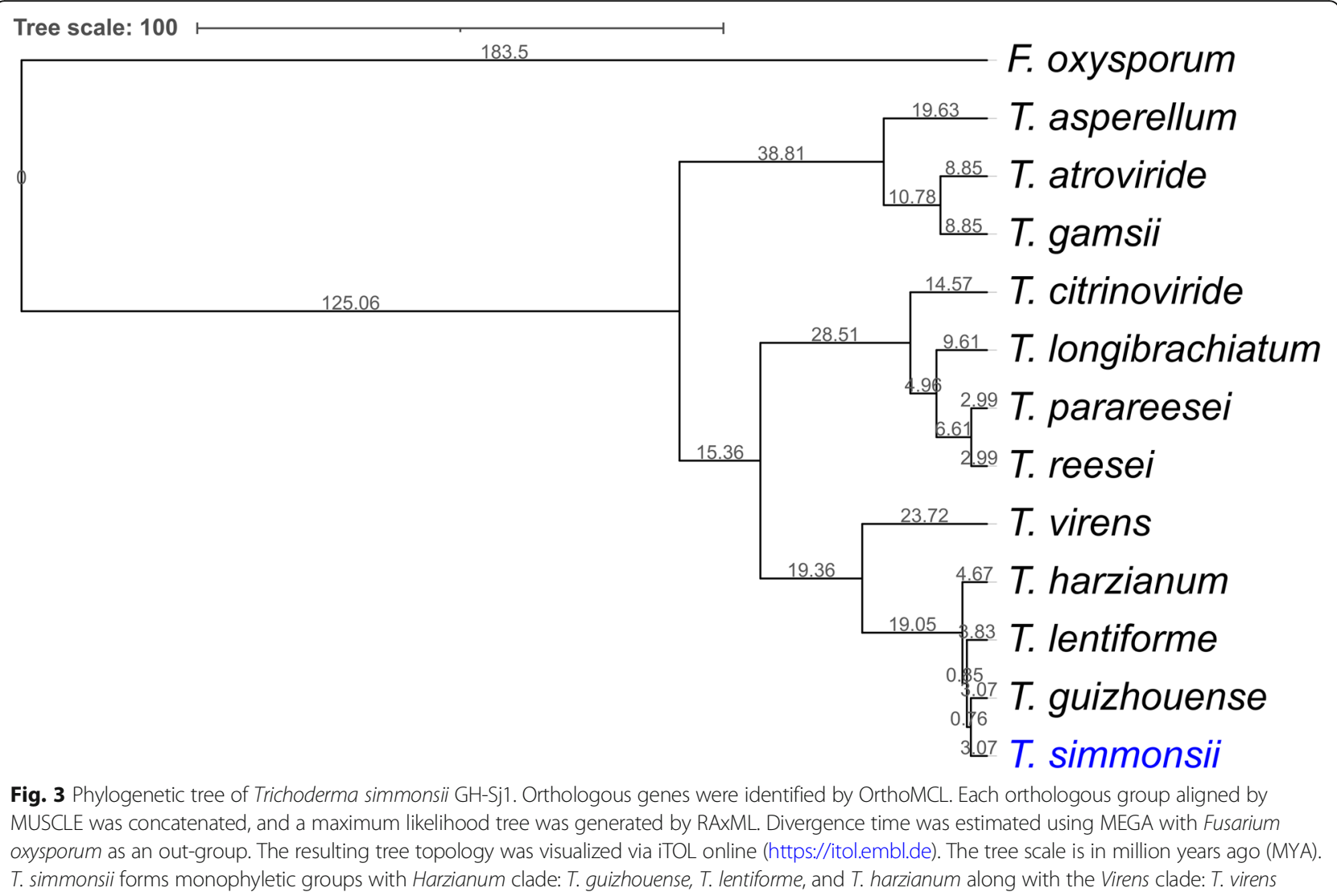

\section{Comparative analyses}

Although the genome structure and composition of $T$. simmonsii was not drastically distinct from those of other Trichoderma spp., we wondered whether Trichoderma simmonsii has a common or unique profile of certain groups of predicted proteins. To address this, various comparative genomic analyses were performed using the funannotate fungal genome analysis suite [29], including comparisons in MEROPS protease families [35], CAZyme families, secreted proteins, and fungal transcription factors. Overall, the distribution of search results from CAZyme, MEROPS, secreted proteins, and fungal transcription factors was similar (Supplementary Figs. 2, 3, 4, 5, 6 and 7) for all Trichoderma spp. analyzed. For all protein family searches, protein-coding genes were most abundant in the $T$. simmonsii genome. In addition, we applied the CAFE program [36] to detect rapidly evolving families of Trichoderma genomes (Supplementary Fig. 8). T. simmonsii had 73 rapidly evolving orthologous gene families, second only to $T$. reesei, which had 94 . In $T$. simmonsii, 72 were from expanded gene families, and one was from a contracted gene family, whereas only 6 were from expanded families, and 88 were from contracted families in $T$. reesei (Supplementary Table 5). T. harzianum had 40 rapidly expanded gene families, second only to T. simmonsii. An asparaginase-related gene, H0G86_011897, which included the PFAM domain of PF01112, was detected in the rapidly expanded gene families of $T$. simmonsii. The full list of rapidly evolving protein families in T. simmonsii is provided in Supplementary Table 6.

\section{RNA-sequencing of $T$. simmonsii isolate GH-Sj1}

While we screened marine fungi for asparaginase activity, results of the phenol red plate assay indicated that GH-Sj1 produced asparaginase. Because Trichoderma spp. are well-known resources for industrial enzyme production $[2,3]$, we selected $\mathrm{GH}-\mathrm{Sj} 1$ for transcriptome analysis of genes possibly related to the asparaginase activity.

To perform RNA-sequencing analysis, first, we investigated asparaginase-rich conditions for fungal cultivation based on the results of phenol-red plate assay. GH-Sj1 was cultivated grew on media containing phenol red with or without L-asparagine (Fig. 4A). The color of phenol red is yellow at $\mathrm{pH} 6.4$ or below, becomes red at pH 8.2, and changes into pink above pH 8.2 [37]. When $\mathrm{NH}_{3}$ is produced via the hydrolysis of L-asparagine by Lasparaginase, an increase of $\mathrm{pH}$ in the cell culture is observed. When grown with L-asparagine, the background color of the GH-Sj1 colony was pink (Fig. 4A). In contrast, on media without L-asparagine, the background 

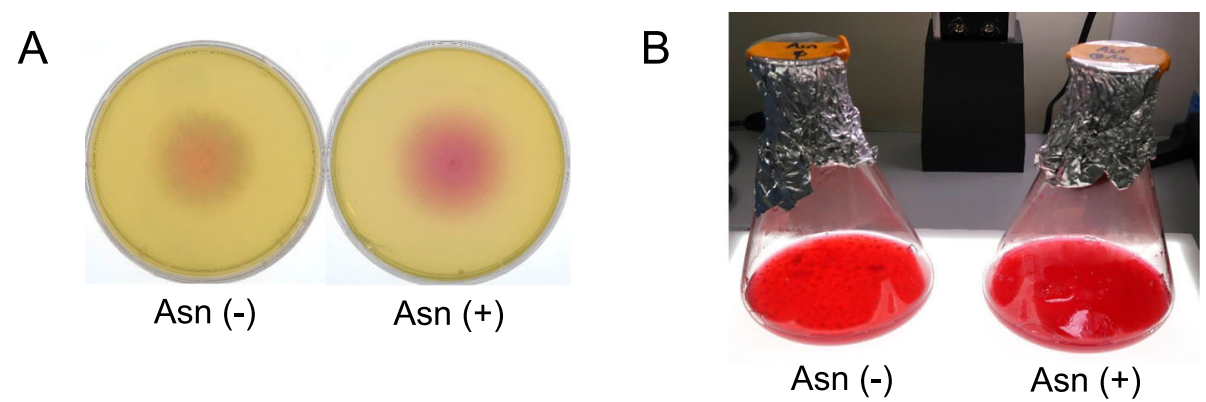

Fig. 4 Asparaginase activity of Trichoderma simmonsii GH-Sj1. GH-Sj1 inoculated on solid media containing 1\% (w/v) asparagine, Asn (+), and no asparagine, Asn (-), as a control. Following incubation at $28^{\circ} \mathrm{C}$ for 3 days, the colony color change (pH change due to asparaginase activity) was observed. (B) Before extracting total RNA, GH-Sj1 was inoculated in liquid media containing $1 \%$ asparagine (asparaginase-rich conditions, Asn (+)) or no asparagine (non-rich conditions, Asn (-)) and cultured at $25^{\circ} \mathrm{C}$ and $200 \mathrm{rpm}$ for 4 days

color of the GH-Sj1 colony was partially reddish or pink, suggesting a more prominent $\mathrm{pH}$ change of the Lasparagine media. This color change was similarly observed in liquid culture of GH-Sj1 for total RNA extraction (Fig. 4B). Therefore, we concluded that addition of $\mathrm{L}$-asparagine to the media resulted in asparaginase-rich conditions of this strain. The media without Lasparagine was used as control conditions.

For transcriptome analysis, both control and experimental samples had two biological replicates: Czp1 and Czp2 for asparaginase non-rich conditions (control); G3 and G4 for asparaginase-rich conditions (experimental samples). RNA sequences for the four samples were generated using the Illumina platform, with sequencing yields of 7,652,661,728 bp from 37,884,464 PE reads, $5,576,060,722$ bp from 27,604,261 PE reads, $7,693,022,136 \mathrm{bp}$ from 38,084,268 PE reads, and 6,590,780,250 bp from 32,627,625 PE reads, respectively. After trimming low-quality bases, 35,271,981 PE reads (7,109,693,702 bp), 25,842,265 PE reads $(5,209,032,962$ bp), 35,517,705 PE reads $(7,158,679,180 \mathrm{bp})$ and $30,568,962$ PE reads $(6,161,876,011 \mathrm{bp})$ remained, respectively.

\section{Putative asparaginase genes in $T$. simmonsii genome}

We performed sequence homology and PFAM asparaginase-related domain searches to uncover genes potentially responsible for the asparaginase activity of GH-Sj1. In total, seven genes in the T. simmonsii genome were identified as putative asparaginase-encoding genes: H0G86_001521, H0G86_011897, H0G86_011901, H0G86_012090, H0G86_012144, H0G86_H012728, and H0G86_H013185. The size of amino acids, gene expression levels, and closely related asparaginase-producing fungal species were listed in Table 5. H0G86_011901 does not include a PFAM asparaginase-related domain but was annotated as "putative L-asparaginase" based on the homology search. Six other genes carried at least one
PFAM asparaginase domain. Based on the types of PFAM domains(s), 6 asparaginase genes in T. simmonsii were classified in 3 categories, as shown in Fig. 5. The majority of genes belonged to Class I, containing the PFAM domain PF01112 (Name: Aspraginase_2; Description: Asparaginase): H0G86_001521, H0G86_011897, H0G86_012090, and H0G86_012144. The E-values of gene pairs measured by BLASTP were between $1.15 \mathrm{e}-05$ and 5.99e-29 (Supplementary Fig. 9). Class II (H0G86_ 012728) contains two asparaginase PFAM domains, PF00710 (Name: Asparaginase; Description: Asparaginase, N-terminal) and PF17763 (Name: Asparaginase_C; Description: Glutaminase/Asparaginase C-terminal domain). Class III (H0G86_013185) contains PFAM domain PF06089 (Name: Asparaginase_II, Description: Lasparaginase II) [38]. Both H0G86_012728 (Class II) and H0G86_013185 (Class III) were dissimilar among other types of asparaginase, with the lowest E-values being 0.22 and 3.0, respectively (Supplementary Fig. 9).

We further investigated the abundances of the three classes of asparaginases in other 11 Trichoderma genomes (Supplementary Table 7). The abundance of Class I asparaginase was $2-4$ in all the genomes. In case of Class II asparaginase, every genome carried a single copy. Similarly, there was a single copy of Class III asparaginase in all genomes except for T. citrinoviride and T. longibrachiatum where PFAM domain PF06089 was not found. In addition, we constructed an asparaginase gene tree. Supplementary Fig. 10 shows phylogenetic relationships of the six asparaginase genes in $T$. simmonsii among other Trichoderma genomes. As with the genome tree, RAxML was used to generate the maximum likelihood gene tree. Each gene group, the tree topology was similar to genome tree, where $T$. simmonsii formed a monophyletic to $T$. guizhouses, $T$. lentiforme, $T$. harzianum and $T$. virens. In case of H0G86_011897, only two neighbors existed which from T. lengtiforme and T. guizhouense. 


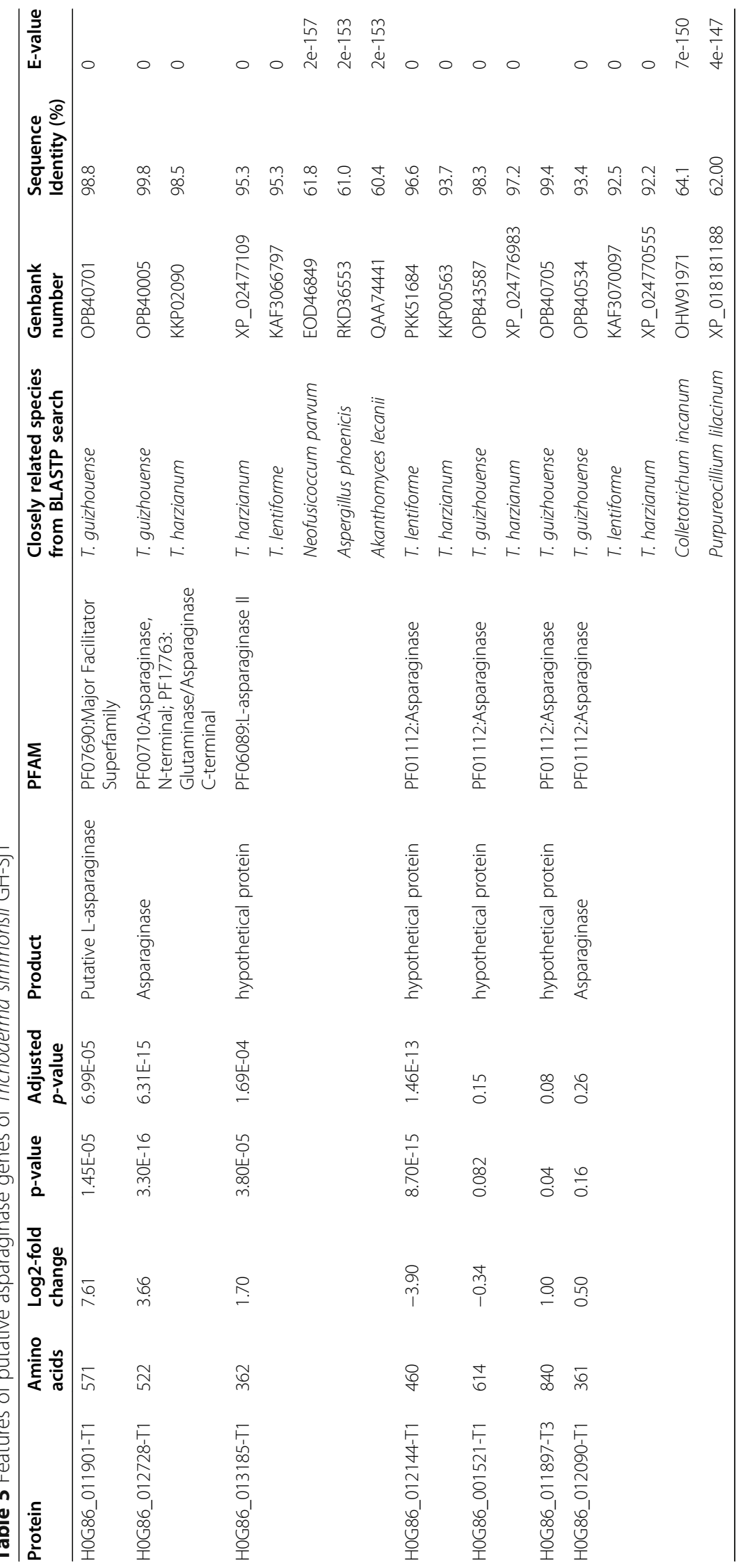




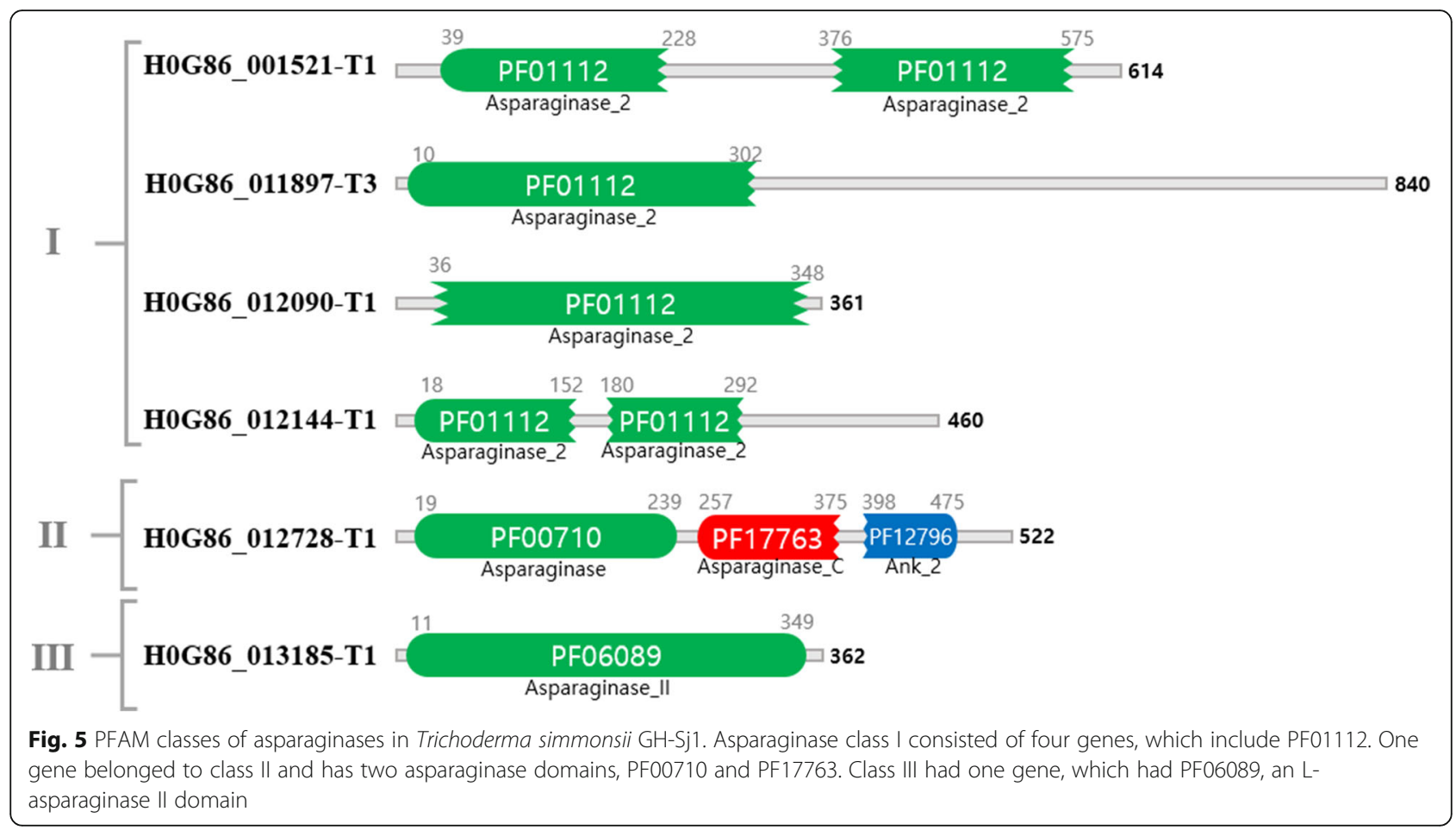

\section{Transcriptional profiles of $T$. simmonsii genes in asparaginase-rich conditions}

Following identification of the putative asparaginase genes, we investigated the overall transcriptional profile of $\mathrm{GH}-\mathrm{Sj} 1$ genes in asparaginase-rich conditions relative to the control. After filtering very lowexpressed transcripts, 12,165 genes were statistically tested using deseq2. Differentially expressed genes (DEGs) were defined as having an expression change of more than 4-fold under asparaginase-rich conditions relative to non-rich conditions. Among these, 5381 genes were differentially expressed (adjusted $P$-value $<=$ 0.05 ) between the two conditions. Approximately $75 \%$ of the genes (3997 genes out of 5381 genes) exhibited functions that could be predicted, and the rest were related to hypothetical proteins. In total, 1384 genes exhibited a 4-fold or greater transcript level change under asparaginase-rich conditions relative to non-rich conditions. Among these, 591 and 793 genes were up- and down-regulated, respectively. Interestingly, the expression of many genes for functions involving glycoside hydrolase were downregulated. For example, endo-1,3(4)- $\beta$-glucanase, endo-1,4- $\beta$-xylanase, chitinases, $\alpha$-galactosidase, and exo- $\beta-1,3$-glucanase exhibited decreased transcript levels in asparaginase-rich conditions.

Genes with increased transcript levels under asparaginase-rich conditions compared to non-rich conditions were enriched in the COG categories of (S) Function unknown (33.7\%), (E) Amino acid transport and metabolism (8.9\%), (Q) Secondary metabolites biosynthesis, transport, and catabolism (7.5\%), (G) Carbohydrate transport and metabolism (7.3\%), (O) Post-translational modification, protein turnover, chaperones $(6.3 \%)$, and $(C)$ Energy production and conversion (5.8\%). Genes with decreased transcript levels were enriched in (S) Function unknown (36.0\%), (G) Carbohydrate transport and metabolism (16.1\%), (E) Amino acid transport and metabolism (8.3\%), (Q) Secondary metabolites biosynthesis, transport and catabolism (7.2\%), (O) Post-translational modification, protein turnover, chaperones $(6.6 \%)$, and $(\mathrm{P})$ Inorganic ion transport and metabolism (5.0\%).

Among the 7 putative asparaginase genes found in our genome analysis of T. simmonsii, 3 (H0G86_011901, H0G86_012728, and H0G86_013185) were up-regulated and 1 (H0G86_012144) was down-regulated under asparaginase-rich conditions compared to non-rich conditions. The most dramatic expression change in response to asparaginase-rich conditions was observed in H0G86_011901. The transcript level of H0G86_011901 increased in asparaginase-rich conditions approximately 128-fold, while those of H0G86_012728 and H0G86 013185 increased approximately 12.6 - and 3.2-fold, respectively. In contrast, the transcript level of H0G86 012144 decreased approximately 15-fold under asparaginase-rich conditions. The expression of three other genes, including H0G86_001521, H0G86_011897, and H0G86_012090, was not significantly affected by asparaginase-rich conditions. 


\section{Discussion}

Trichoderma species have been widely used as biocontrol agents and producers of industrial enzymes. In this work, we studied marine-derived $T$. simmonsii strain $\mathrm{GH}-\mathrm{Sj} 1$ to understand its genomic structure and transcriptional profiles associated with asparaginase production. T. simmonsii was previously identified as a new species within the Harzianum clade of Trichoderma based on internal transcribed spacer (ITS) and translation elongation factor 1- $\alpha$ (TEF1) sequences [5]. However, relative to other Trichoderma species, T. simmonsii remains largely unknown.

A complete, telomere-to-telomere, chromosome-level reference genome assembly is a valuable resource and essential for studies on chromosome evolution and lineage-specific adaptation by ensuring that all genomic variants are discovered and studied [39, 40]. With the rapid advance in sequencing technologies, more and more researchers are performing assembly at the chromosome-level [41]. Telomere-to-Telomere (T2T) consortium accomplished a complete human X chromosome assembly and ultimately plans to complete, high quality telomere-to-telomere assemblies from diploid human genomes [40]. In case of larger brewing yeast Saccharomyces pastorianus strains, the existing incomplete and highly fragmented genome assembly was improved on chromosome-scale assembly using Oxford Nanopore MinION sequencing [42]. In addition, Trichoderma reesei QM6a genome achieved chromosome-level assembly by resequencing using $\mathrm{PacBio}$ and $\mathrm{Hi}-\mathrm{C}$ technologies [27]. In this study, we accomplished telomereto-telomere assembly of $T$. simmonsii genome using only Oxford Nanopore long reads and Illumina PE reads. We demonstrated that a high quality fungal genome was assembled by comparing and carefully curating outputs from multiple de novo assemblies without reference to existing assemblies or long range contact information from $\mathrm{Hi}-\mathrm{C}$.

The assembly quality of $T$. simmonsii genome was 98.7\% with a BUSCO value despite a complete genome. Similarly, for complete genomes $S$. pastrorianus and $T$. reesei, BUSCOs were 90.0 and $99.2 \%$, respectively. A chromosome-level genome assembly does not necessarily guarantee a complete BUSCO score. It may be because the assembly is not $100 \%$ accurate, but at the same time, the BUSCO value may not be a perfect indicator of genomic quality assessment. As discussed in the paper, the BUSCO assessment fine-tuned the parameters of score and length cutoff to maximize accuracy, but may still fall short of $100 \%$ accurate results as both genetic prediction and orthology assignment are challenging tasks, which can be resulted in missing or incorrect BUSCO predictions [43]. This limitation was also observed in the paper where low BUSCO scores could be caused by missing genes in the gene prediction step of the tool [44]. Despite its limitations, without a definitive alternative, BUSCO is still an essential genomic quality assessment tool that includes up-to-date data from many species. Through the identification of orthologs in the genomes of 12 Trichoderma species, we demonstrated that $T$. simmonsii was grouped with fungi belonging to the Harzianum clade, in agreement with this previous report.

Overall, the Harzianum/Virens clade had more rapidly expanded than rapidly contracted gene families, with $T$. simmonsii having the most expanded families within the clade. Gene family expansion is known to provide a significant evolutionary boost wherein selection may play a role in promoting adaptation [45]. Further, rapid gene family expansion is associated with adaptive natural selection in favor of additional copies either in order to increase dosage or the arsenal of molecular weaponry [46]. T. reesei and T. parareesei of the Longibrachiatum clade had similar rapidly evolving gene families to $T$. simmonsii, but the majority were contracted gene families.

For the transcriptome analysis, we focused on asparaginase production of GH-Sj1. Asparaginase is an enzyme used to treat leukemia and to reduce carcinogenic compounds in food $[14,15]$. Currently, bacterial asparaginases are mostly used while fungal asparaginases have been poorly characterized, in particular, at the genomic levels. For example, based on data from the National Center for Biotechnology Information (NCBI), the majority of reported L-asparaginase protein sequences originated from bacteria (95.5\%). Fungal L-asparaginase sequences account for only approximately $1.7 \%$ [15]. Lasparaginase-producing fungal species are represented by those belonging to the Aspergillus, Penicillium, Fusarium, Cladosporium, and Trichoderma genera [15]. Moreover, two types of L-asparaginase and related genes have been identified in Saccharomyces cerevisiae (baker's yeast) [47].

Asparaginase activity of GH-Sj1 was examined using media containing a $\mathrm{pH}$ indicator phenol red. Although this method has been widely used for screening microorganisms with asparaginase activity [48], it is noteworthy that positive results from this assay require additional analyses (enzyme purification, etc.) to confirm asparaginase production of the microorganisms. Although the degree of color change ( $\mathrm{pH}$ change) was more significant when asparagine was added to the media, we observed that growth of $\mathrm{GH}-\mathrm{Sj} 1$ caused color change in the absence of asparagine (Fig. 4). This suggests that other factors in addition to hydrolysis of asparagine by asparaginase could result in $\mathrm{pH}$ change in media. Due to this limitation, sometimes media to screen of asparaginase activity could be optimized by changing carbon sources or concentrations of salts and phenol red [49]. 
Through the results of genome analysis, we identified seven asparaginase-related genes in T. simmonsii GHSj1. As previously described, H0G86_011901, which was included based solely on strong sequence homology, exhibited the greatest up-regulation under asparaginaserich conditions. However, without molecular cloning and enzyme purification, it is currently difficult to conclude which gene(s) are responsible for the asparaginase activity of T. simmonsii GH-Sj1 under the tested conditions. In order to elucidate the link between genes and asparaginase activity, future studies will include the generation and characterization of null or overexpression mutants for each gene. Furthermore, it should be noted that the transcriptional profiles were investigated at a single time point. Therefore, the expression levels of each gene over the distinct incubation time could give us further insight into its role in asparaginase activity.

\section{Conclusions}

In this study, we sequenced T. simmonsii GH-Sj1, which was isolated from sea algae Saccharina japonica, using both short and long read platforms. The chromosomescale $T$. simmonsii genome was obtained through comparing multiple long read assemblers and manual curation. The resulting genome consisted of seven telomere-to-telomere scaffolds with no gaps. The assembled genome was $\sim 40 \mathrm{Mb}$ in length and had a GC content of $48.13 \%$. The genome completeness of T. simmonsii was $\sim 99 \%$. The T. simmonsii genome harbored 13,120 protein-coding genes, 176 tRNAs, and an rRNA repeat region, which consisted of seven repeats of the 18S-ITS1-5.8S-ITS2-26S cluster. Through a close homology search and PFAM domain search, seven putative asparaginase-related genes were identified, of which three were up-regulated under asparaginase-rich conditions. To our best of knowledge, this is the first report of the $T$. simmonsii genome, thus representing a valuable resource for the further study of enzymatic activities, including that of asparaginase, as well as comparative studies of fungal genomes.

\section{Methods}

\section{Sample collection and fungal isolation}

Sea algae, Saccharina japonica, also called as Kombu, was collected from Gul-Hang Quay at Sacheon, Gyeongsangnamdo Province, Republic of Korea $\left(34.55^{\prime} 43.5^{\prime \prime} \mathrm{N}\right.$, $\left.128.03^{\prime} 24.8^{\prime \prime} \mathrm{E}\right)$. It was washed with sterile water, cut into about $1-\mathrm{cm}$ segments using sterile scissors, and placed onto potato dextrose agar (PDA; BD) and yeastmold agar (YM agar; BD) containing $0.01 \%(w / v)$ ampicillin and $0.01 \%(\mathrm{w} / \mathrm{v})$ streptomycin. After incubation at $20^{\circ} \mathrm{C}$ for 14 days, fungal colonies were isolated and transferred to fresh PDA until pure spores were obtained. The fungal isolates were stored in $20 \%$ glycerol solution at $-80^{\circ} \mathrm{C}$ and deposited in the National Marine Biodiversity Institute of Korea (MABIK).

\section{Morphological characterization of GH-Sj1}

Following growth on PDA at $25^{\circ} \mathrm{C}$ for 7 days, GH-Sj1 colony morphology was observed and conidia were collected using sterile $\mathrm{H}_{2} \mathrm{O}$. Conidia and conidiophore morphology were observed using a Leica CTR6000 microscope equipped with a Leica DMC2900 camera (Leica, Germany). Image acquisition and processing were performed using LASV4.5 software (Leica).

\section{Genomic DNA extraction}

Extraction of fungal genomic DNA was performed as previously described [50]. Briefly, fungal isolates were cultured in potato dextrose broth (PDB; BD) at $25^{\circ} \mathrm{C}$, $200 \mathrm{rpm}$ for 3 days. Mycelia were harvested using Miracloth (Millipore), frozen using liquid nitrogen, and ground with a mortar and a pestle. The ground fungal tissue was suspended using lysis buffer followed by addition of phenol: chloroform: isoamyl alcohol (25:24:1) (Sigma, US). After centrifugation at $4{ }^{\circ} \mathrm{C}$ and $13,000 \mathrm{rpm}$ for $10 \mathrm{~min}$, the aqueous layer was collected, and genomic DNA was precipitated via the addition of isopropanol. DNA was harvested by centrifugation at $13,000 \mathrm{rpm}$ for $5 \mathrm{~min}$, dried at room temperature, and dissolved with nuclease-free water.

\section{PCR and fungal identification}

For the molecular identification of fungal strain $\mathrm{GH}-\mathrm{Sj} 1$, polymerase chain reaction (PCR) was performed using primers EF1-728F [17] and TEF1LLErev [18] in order to amplify tef1 $\alpha$, which encodes translation elongation factor $1 \alpha$. PCR running conditions were as follows: $2 \mathrm{~min}$ at $94{ }^{\circ} \mathrm{C}$; 35 cycles of $30 \mathrm{~s}$ at $94^{\circ} \mathrm{C}, 30 \mathrm{~s}$ at $55^{\circ} \mathrm{C}$, and 1 min at $72{ }^{\circ} \mathrm{C}$, and, finally, $72{ }^{\circ} \mathrm{C}$ for $15 \mathrm{~min}$. Purification was then performed using a QIAquick PCR Purification Kit (Qiagen, Germany), and the sequences of PCR products were analyzed by Macrogen (Macrogen, Korea). The obtained sequences of tefl $\alpha$ were used to search closely related species in GenBank via BLASTN [51].

\section{Examination of asparaginase activity}

Fungal isolates were cultured on Czapek-Dox broth $(\mathrm{CDB}, \mathrm{BD})$ supplemented with $0.1 \%(\mathrm{w} / \mathrm{v})$ yeast extract, $1 \%(\mathrm{w} / \mathrm{v})$ L-asparagine monohydrate (Sigma), and $0.005 \%(\mathrm{w} / \mathrm{v})$ phenol red (Sigma). As a control, the isolates were cultured on the same media without Lasparagine monohydrate. The $\mathrm{pH}$ values of all media were adjusted to $\mathrm{pH}$ 6.0. To obtain fungal spores, we cultured GH-Sj1 on PDA at $25^{\circ} \mathrm{C}$ for 7 days, and collected spores using sterile $\mathrm{H}_{2} \mathrm{O}$. Five microliters of the spore suspension were inoculated in the center of the phenol red plates. After incubation at $25^{\circ} \mathrm{C}$ for 3 days, 
the color of the medium was observed. Compared to the control plate, the color change from orange to pink in the media containing L-asparaginase was considered to indicate asparaginase activity of the tested strain.

\section{Total RNA extraction}

GH-Sj1 $\left(5 \times 10^{7}\right.$ conidia $)$ was cultured in $100 \mathrm{ml}$ of Czapek-Dox broth supplemented with $0.1 \%(\mathrm{w} / \mathrm{v})$ yeast extract and 1\% (w/v) L-asparagine monohydrate (asparaginase-rich conditions) or without L-asparagine monohydrate (non-rich conditions). Following incubation at $25^{\circ} \mathrm{C}$ and $200 \mathrm{rpm}$ for 4 days, mycelia were harvested, flash frozen in liquid nitrogen, and ground in a mortar. Fungal tissue was suspended in $1 \mathrm{ml}$ TRIzol reagent (Invitrogen, US) followed by the addition of $200 \mu \mathrm{l}$ chloroform (Sigma). After centrifugation at $4{ }^{\circ} \mathrm{C}$ and $13,000 \mathrm{rpm}$ for $15 \mathrm{~min}$, the upper layer was transferred to a fresh microcentrifuge tube. RNA was precipitated with $80 \%$ ethanol and purified using the RNeasy plant mini kit (Qiagen). This experiment was performed in two biological replicates.

\section{DNA library construction and sequencing}

For Illumina sequencing, a sequencing library with short inserts of $550 \mathrm{bp}$ for paired-end reads were prepared using the Truseq DNA PCR-Free kit as per the manufacturer's protocol for Illumina (Illumina, San Diego, CA, USA). $2 \times 151 \mathrm{bp}$ reads were generated on an Illumina NovaSeq6000 platform (Illumina) at DNALink (Seoul, S. Korea). For long read sequencing, a 1D sequencing library was constructed using Oxford Nanopore Technologies' standard ligation sequencing kit SQK-LSK109. FLO-MIN106 (R9.4) flow cells were used for sequencing on the GridION X5 platform (Oxford Nanopore Technologies, Oxford, UK) at MABIK.

\section{RNA library construction and sequencing}

Four sets of sequencing libraries for paired-end reads were prepared using the TruSeq Stranded mRNA kit following the manufacturer's protocol for Illumina (Illumina, San Diego, CA, USA). Products were quantified using the Bioanalyzer 2100 (Agilent, Santa Clara, CA, USA), and $2 \times 101 \mathrm{bp}$ reads were generated on an Illumina NovaSeq6000 platform (Illumina) at DNALink.

\section{Sequence preprocessing}

Illumina WGS reads were quality-trimmed using Trimmomatic (v0.36) [52] with the following trimming options "2:30:10 LEADING:3 TRAILING:3 SLIDINGWINDOW:4:15 MINLEN:75." Similarly, Illumina RNAsequencing reads were quality-trimmed using the same software with identical options except for MINLEN:50. The base calling of Nanopore read bases was performed using guppy (v3.2.10) from Oxford Nanopore Technologies
[53] with default settings, except for high accuracy mode. Prior to the genome assembly, adapter sequences of Nanopore reads were trimmed using PoreChop (v0.2.4) [54].

\section{Genome assembly and polishing}

Nanopore sequencing reads with at least $10 \mathrm{~Kb}$ were assembled using Canu (v1.8) [22], Flye (v2.4) [23], Miniasm assembler (v0.3-r179) [24], Shasta (v0.4.0) [25], and Wtdbg2 (v2.3) [26] assemblers, separately. The initial draft assemblies were polished with adapter-trimmed Nanopore reads of all sizes using MarginPolish (v1.3.dev-5,492,204), followed by HELEN [25]. The polished contigs were further polished 5 times with quality-trimmed Illumina PE reads using Pilon (v1.22) [55]. Genome assembly statistics were calculated from QUAST (v4.5) [56], and the completeness of genome assemblies was evaluated using BUSCO (v4.0.6) [28].

\section{Telomere repeat detection}

Tandem repeat finder (trf v4.04) [57] was used to find all tandem repeats on each contig. We then manually inspected the existence of telomere repeats in each terminus of the contig, namely CCCTAA ( $5^{\prime}$ terminus) and TTAGGG ( 3 ' terminus).

\section{Scaffolding and assembly correction}

Whole-genome pairwise alignment from two different assemblers was performed using chromeister (v0.8) [58], as shown in Supplementary Fig. 11. Based on the assembly results and telomere repeats summarized in Supplementary Table 1, Miniasm was chosen for a baseline draft assembly, as it was in highest continuity, consisting of only nine contigs with $\mathrm{N} 50$ of $6.4 \mathrm{Mb}$ and having the greatest support for telomere ends, with five telomereto-telomere contigs. Seven Miniasm contigs were greater than $1 \mathrm{Mb}$ in length. The remaining two contigs were $28 \mathrm{~Kb}$ and $\sim 5 \mathrm{~Kb}$ in length. The shortest contig $(\sim 5 \mathrm{~Kb})$ was discarded because it was even shorter than the minimum input read length $(10 \mathrm{~Kb})$. The second shortest contig $(\sim 28 \mathrm{~Kb})$ was identified as the mitochondrial genome after aligning it to mitochondrial genomes of related species. Two overlapping contigs $(\sim 3.1 \mathrm{Mb}$ and $\sim 1.5 \mathrm{Mb}$ ), labeled as 5 and 6 in the y-axis in Supplementary Fig. 11, were concatenated because they overlapped more than $50 \mathrm{~Kb}$ in the $3^{\prime}$ and $5^{\prime}$ termini. The two contigs were assembled as single contig in Flye and Wtdbg2 assemblers. In addition, the two contigs had a terminus of either TTAGGG $\left(5^{\prime} \rightarrow 3^{\prime}\right)$ or CCCTAA $\left(3^{\prime} \rightarrow 5^{\prime}\right)$ telomere repeats, but not both. The extended scaffold was polished again using the method mentioned above. The longest contig labeled as 1 in the $y$-axis in the same figure was split to two contigs due to two centromere regions as illustrated in Supplementary Fig. 12. In addition, 
the scaffold was assembled as two contigs in Wtdbg2 assembly (Supplementary Fig. 11 (b)). The longer part was replaced with the corresponding Wtdbg2 contig after confirming that it contained both $5^{\prime}$ and $3^{\prime}$ telomere sequences. On the other hand, the Wtdbg2 contig corresponding to the shorter region did not have telomere repeats. We performed local assembly using Miniasm with $>=10 \mathrm{~Kb}$ Nanopore reads which did not align to other scaffolds by dropping the reads that aligned $>=80 \%$ length to the other scaffolds using minimap2. The longest contigs from the new local assembly were recruited after polishing using the methods described earlier. The total number of scaffolds became seven, and the scaffolds were renamed in decreasing order by lengths. Scaffold ends were adjusted by trimming bases with no short read supports using bwa mem (v0.7.15-r1140) [59] and BEDTools (v2.26.0) [60] via genomeCoverageBed.

\section{Gene prediction and genome annotation}

The genome of $T$. simmonsii was annotated structurally and functionally using funannotate (v1.7.4). The step-bystep pipeline usage is well documented in [61]. Briefly, repeat contains were masked using tantan (v13) [62]. After aligning RNA-sequencing data to the genome using HISAT2 (v2.2.0) [63], genome-guided transcriptome assemblies were generated using Trinity (v2.8.5) [64], followed by PASA (v2.4.1) [65]. Since a fungal genome is expected to have high gene density, the --jaccard_clip option was used for this training stage. Multiple gene models were then predicted by 1 ) aligning protein sequences to UniProt/SwissProt (2020_03) using DIAMOND (v0.9.21) [66] and EXONERATE (v2.4.0) [67]; 2) performing GeneMark-ES (v4.59) [68] in selftraining mode; 3) executing Augustus (v3.3.3) [69], GlimmerHMM (v3.0.4) [70] as well as SNAP (v2013_11_ 29) [71] with PASA hints; and 4) running CodingQuarry (v2.0) [72] with RNA-sequencing alignment. All these outputs were passed into EvidenceModeler (v1.1.1) [73] to select the consensus models among the ab initio and evidence-based gene models. The gene models were filtered based on length cutoff $(<50 \mathrm{bp})$, spanning gaps, and the existence of transposable elements. The tRNA genes were predicted with tRNAscan-SE (v2.0.5) [74]. Using the funannotate update command, UTRs were added to gene models. Various functional features were assigned, such as Phobius (v1.01) [75] results, antiSMASH (v5) [32], eggnog-mapper (v2.0.1b) [76], InterProScan (v5.50-84.0) [77], HMMer (v3.3) [78] search of PFAM (v33.1) [79], CAZymes (dbCAN v8.0) [31] using HMMer, and the Diamond blastp search of MEROPS (v12.0) [35].

Apart from the funannotate pipeline, protein functions (i.e., product field) were revised in the following manner. Protein sequences were aligned with BLASTP against all dikarya protein sequences in UniProt DB (v2021_03). Matching sequences were kept when the E-value was <= $1.0 \mathrm{e}-10$, percent identity $>=50 \%$, and query coverage in alignment $>=50 \%$. Protein function was taken from the top hit. When more than one protein sequences from the same gene had a different functional description, we manually corrected them to have same functional description.

\section{Circular genome map}

A circular genome map was drawn using ShinyCircos [80]. From outside to inside, the first ring shows the locus of scaffolds. The second and third rings are mapping coverages of Illumina and Nanopore reads. Illumina DNA reads were aligned using bwa mem with default parameters. For Nanopore reads, minimap2 was used with -x map-ont -a options. The resulting bwa and minimap2 BAM files were sorted in genomic locus order, and base coverage was calculated using genomeCoverageBed with - $d$ option. Average coverage in a $1 \mathrm{~Kb}$ window was measured, and the $\log 2$ of average coverage was used for efficient coverage plotting due to the existence of several very high-coverage regions which made other regions indistinguishable. The fourth ring is the $\mathrm{GC}$ content line which was also drawn in the $1 \mathrm{~Kb}$ window. The fifth ring is the gene counts in the $100 \mathrm{~Kb}$ window plotted as bar charts. The track and names above the title are the loci of asparaginase-related genes.

\section{Comparative genomics}

In order to perform comparative analyses of T. simmonsii to 11 reference genomes under equivalent conditions, the reference genomes were re-annotated using funannotate. Funannotate was then used to perform comparative analyses of functional categories such as PFAM, InterProScan, CAZyme, MEROPS, secreted proteins, and fungal transcription factors.

\section{Orthology and phylogeny}

Orthologous protein sequences of T. simmonsii and 12 reference genomes were identified using OrthoMCL (v2.0.9) [81]. Orthologous group consisting of single protein sequences across all genomes were aligned using MUSCLE (v3.8.31) [82] with default options. After concatenation of all orthologous groups, gap regions were trimmed using trimAl (v1.4.rev6) [83] with the -phylip -gappyout option. A maximum likelihood tree was generated using RAxML (v8.2.10) [33] with the following options: -m PROTGAMMAJTT -x 12,345 -p 12345 -N 100 -f a $-\mathrm{T}$ 8. Divergence times in the tree were estimated using MEGA (v7.0) [34] with -O F. oxysporum -C 'T. harzianum F. oxysporum 98269' options. F. oxysporum was assigned as an outgroup, and the time interval between $F$. oxysporum and $T$. harzianum $(98,269)$ 
taken from TimeTree [84] was used for branching calculation. CAFE (v4.2.1) [36] was used to identify rapidly evolving families by inputting OrthoMCL output and divergence time estimated from MEGA.

\section{DEGs}

Four RNA read sets (two controls and two experiments) were aligned against the transcriptome using Salmon aligner (v1.4.0) [85]: i.e. salmon quant with -l A --validateMappings options. The mapping results were loaded to deseq2 [86] using tximport function on $\mathrm{R}$ (v3.6.0) [87]. Genes expressed at a very low level were removed when the maximum mapping count of each group's median value was below 10. Subsequent DEG analyses were performed as per the deseq2 manual. Genes whose transcript levels changed 4-fold or greater were included as DEGs.

\section{Abbreviations}

bp: base pair; CAZyme: Carbohydrate-active enzymes; COG: Clusters of orthologous groups of proteins; DEG: Differentially expressed gene; Gb: Gigabase; GO: Gene ontology; ITS: Internal transcribed spacer; Kb: Kilobase; MABIK: National Marine Biodiversity Institute of Korea; Mb: Megabase; MYA: Million years ago; NCBI: National Center for Biotechnology Information; PCR: Polymerase chain reaction; PE: Paired-end; RNA: Ribonucleic acid; rRNA: ribosomal ribonucleic acid; tRNA: transfer ribonucleic acid

\section{Supplementary Information}

The online version contains supplementary material available at https://doi. org/10.1186/s12864-021-08162-4.

\section{Additional file 1 \\ Additional file 2. \\ Additional file 3 .}

\section{Acknowledgements}

The authors also wish to thank Dr. Kyungwha Baek for critically reading the manuscript and the helpful discussion. We would like to thank Editage (www.editage.co.kr) for English language editing.

\section{Authors' contributions}

YY designed the project. YMK and DC isolated samples. Experiments performed by DC and YY. YY conducted bioinformatics analyses. DC helped the interpretation of the transcriptome result. DC and $Y Y$ wrote the manuscript. YMK reviewed and edited the manuscript. All authors read and approved the final manuscript.

\section{Funding}

This work was supported by the MABIK in-house programs (2021M00500 and 2021M00600).

\section{Availability of data and materials}

This project has been deposited in NCBI under BioProject accession PRJNA645793. BioSample accessions for WGS and four RNA samples are SAMN15516371, SAMN19276232, SAMN19276234, SAMN19276235, and SAMN19276236. Raw sequence reads have been deposited in the SRA under accession number SRR14597877 through SRR14597884. The locus tag of $T$. simmonsii is H0G86 and the NCBI accession numbers for seven scaffolds are CP075864 through CP075870. All data is publicly available from NCBI and can be accessed at https://www.ncbi.nlm.nih.gov/bioproject/PRJNA645793.

\section{Declarations}

Ethics approval and consent to participate

Not applicable.

Consent for publication

Not applicable.

\section{Competing interests}

The authors declare that they have no competing interests.

Received: 2 August 2021 Accepted: 2 November 2021

Published online: 17 November 2021

\section{References}

1. di Cologna NM, Gómez-Mendoza DP, Zanoelo FF, Giannesi GC, Guimarães NCD, Moreira LRD, et al. Exploring Trichoderma and aspergillus secretomes: proteomics approaches for the identification of enzymes of biotechnological interest. Enzym Microb Technol. 2018;109:1-10. https://doi. org/10.1016/j.enzmictec.2017.08.007.

2. Bischof RH, Ramoni J, Seiboth B. Cellulases and beyond: the first 70 years of the enzyme producer Trichoderma reesei. Microb Cell Factories. 2016;15(1): 106. https://doi.org/10.1186/s12934-016-0507-6.

3. Merck KgaA, Damstadt, Germany. 2021. https://www.sigmaaldrich.com.

4. Fraceto LF, Maruyama CR, Guilger M, Mishra S, Keswani $C$, Singh HB, et al. Trichoderma harzianum-based novel formulations: potential applications for management of next-gen agricultural challenges. J Chem Technol Biotechnol. 2018;93(8):2056-63. https://doi.org/10.1002/jctb.5613.

5. Chaverri P, Branco-Rocha F, Jaklitsch W, Gazis R, Degenkolb T, Samuels GJ. Systematics of the Trichoderma harzianum species complex and the reidentification of commercial biocontrol strains. Mycologia. 2015;107(3):55890. https://doi.org/10.3852/14-147.

6. Gu X, Wang R, Sun Q, Wu B, Sun JZ. Four new species of Trichoderma in the Harzianum clade from northern China. MycoKeys. 2020;73:109-32. https:// doi.org/10.3897/mycokeys.73.51424.

7. Liang $X R, M a X Y$, Ji NY, Trichosordarin A. A norditerpene glycoside from the marine-derived fungus Trichoderma harzianum R5. Nat Prod Res. 2019; 34(14):2037-42. https://doi.org/10.1080/14786419.2019.1574782.

8. Tang R, Kimishima A, Ishida R, Setiawan A, Arai M. Trichosordarin a, a norditerpene glycoside from the marine-derived fungus Trichoderma harzianum R5. J Nat Med. 2020;74(1):153-8. https://doi.org/10.1080/1478641 9.2019.1574782

9. Qiao M, Du X, Zhang Z, Xu J, Yu Z. Three new species of soil-inhabiting Trichoderma from Southwest China. MycoKeys. 2018:44(44):63-80. https:// doi.org/10.3897/mycokeys.44.30295.

10. Kim K, Heo YM, Jang S, Lee H, Kwon S-L, Park MS, et al. Diversity of Trichoderma spp. in marine environments and their biological potential for sustainable industrial applications. Sustainability. 2020;12(1):4327. https://doi. org/10.3390/su12104327.

11. Yaghoubian $Y$, Siadat SA, Telavat MRM, Pirdashti H, Yaghoubian I. Bioremoval of cadmium from aqueous solutions by filamentous fungi: Trichoderma spp. and Piriformospora indica. Env Sci Pollut Res Int. 2019;26(8): 7863-72. https://doi.org/10.1007/s11356-019-04255-6.

12. Priest SJ, Yadav V, Heitman J. Advances in understanding the evolution of fungal genome architecture. F1000Res. 2020;9:776. https://doi.org/10.12688/ f1000research.25424.1.

13. Kubicek CP, Steindorff AS, Chenthamara K, Manganiello G, Henrissat B, Zhang J, et al. Evolution and comparative genomics of the most common Trichoderma species. BMC Genomics. 2019;20(1):485. https://doi.org/10.1186/ s12864-019-5680-7.

14. Ramya LN, Doble M, Rekha VP, Pulicherla KK. L-Asparaginase as potent antileukemic agent and its significance of having reduced glutaminase side activity for better treatment of acute lymphoblastic leukaemia. Appl Biochem Biotechnol. 2012;167(8):2144-59. https://doi.org/10.1007/s12010012-9755-z.

15. da Cunha MC, Aguilar JGDS, de Melo RR, Nagamatsu ST, Ali F, de Castro RJS, et al. Fungal L-asparaginase: strategies for production and food applications, Food Res Int. 2019;126:108658. https://doi.org/10.1016/j.foodres.2019.108658.

16. Cachumba JJ, Antunes FA, Peres GF, Brumano LP, Santos JC, da Silva SS. Current applications and different approaches for microbial l-asparaginase 
production. Braz J Microbiol. 2016;47:77-85. https://doi.org/10.1016/j.bjm.2 016.10.004.

17. Carbone I, Kohn LM. A method for designing primer sets for speciation studies in filamentous ascomycetes. Mycologia. 1999;91(3):553-6. https://doi. org/10.2307/3761358.

18. Jaklitsch WM, Komon M, Kubicek CP, Druzhinina IS. Hypocrea vogImayrii sp. nov. from the Austrian Alps represents a new phylogenetic clade in Hypocrea/Trichoderma. Mycologia. 2005;97(6):1365-78. https://doi.org/10.3 852/mycologia.97.6.1365

19. Goodwin S, McPherson JD, McCombie WR. Coming of age: ten years of next-generation sequencing technologies. Nat Rev Genet. 2016;17(6):33351. https://doi.org/10.1038/nrg.2016.49.

20. Pop M, Salzberg SL. Bioinformatics challenges of new sequencing technology. Trends Genet. 2008;24(3):142-9. https://doi.org/10.1016/j.tig.2 007.12.006.

21. Klassen $\mathrm{J}$, Currie CR. Gene fragmentation in bacterial draft genomes: extent, consequences and mitigation. BMC Genomics. 2012;13(1):14. https:// doi.org/10.1186/1471-2164-13-14.

22. Koren S, Walenz BP, Berlin K, Miller JR, Bergman NH, Phillippy AM. Canu: scalable and accurate long-read assembly via adaptive $\mathrm{k}$-mer weighting and repeat separation. Genome Res. 2017;27(5):722-36. https://doi.org/10.1101/ gr.215087.116.

23. Kolmogorov M, Yuan J, Lin Y, Pevzner PA. Assembly of long, error-prone reads using repeat graphs. Nat Biotechnol. 2019;37(5):540-6. https://doi. org/10.1038/s41587-019-0072-8.

24. Li H. Minimap and miniasm: fast mapping and de novo assembly for noisy long sequences. Bioinformatics. 2016;32(14):2103-10. https://doi.org/10.1 093/bioinformatics/btw152.

25. Shafin $\mathrm{K}$, Pesout T, Lorig-Roach R, Haukness M, Olsen HE, Bosworth C, et al. Nanopore sequencing and the Shasta toolkit enable efficient de novo assembly of eleven human genomes. Nat Biotechnol. 2020;38(9):1044-53. https://doi.org/10.1038/s41587-020-0503-6.

26. Ruan J, Li H. Fast and accurate long-read assembly with wtdbg2. Nat Methods. 2020;17(2):155-8. https://doi.org/10.1038/s41592-019-0669-3.

27. Li WC, Huang CH, Chen CL, Chuang YC, Tung SY, Wang TF. Trichoderma reesei complete genome sequence, repeat-induced point mutation, and partitioning of CAZyme gene clusters. Biotechnol Biofuels. 2017;10(1):170. https://doi.org/10.1186/s13068-017-0825-x.

28. Simão FA, Waterhouse RM, loannidis $P$, Kriventseva EV, Zdobnov EM. BUSCO: assessing genome assembly and annotation completeness with single-copy orthologs. Bioinformatics. 2015;31 (19):3210-2. https://doi.org/1 0.1093/bioinformatics/btv351.

29. GitHub, Inc. Funannotate. 2021. https:/github.com/nextgenusfs/funannotate.

30. Tatusov RL, Galperin MY, Natale DA, Koonin EV. The COG database: a tool for genome-scale analysis of protein functions and evolution. Nucleic Acids Res. 2000;28(1):33-6. https://doi.org/10.1093/nar/28.1.33.

31. Lombard V, Ramulu HG, Drula E, Coutinho PM, Henrissat B. The carbohydrate-active enzymes database (CAZy) in 2013. Nucleic Acids Res. 2014;42(Database issue):D490-5. https://doi.org/10.1093/nar/gkt1178.

32. Medema MH, Blin K, Cimermancic P, de Jager V, Zakzewski P, Fischbach MA, et al. antiSMASH: rapid identification, annotation and analysis of secondary metabolite biosynthesis gene clusters in bacterial and fungal genome sequences. Nucleic Acids Res. 2011;39(Web Server issue):W339-W46. https:// doi.org/10.1093/nar/gkr466.

33. Stamatakis A. RAxML version 8: a tool for phylogenetic analysis and postanalysis of large phylogenies. Bioinformatics. 2014;30(9):1312-3. https://doi. org/10.1093/bioinformatics/btu033.

34. Tamura K, Peterson D, Peterson N, Stecher G, Nei M, Kumar S. MEGA5: molecular evolutionary genetics analysis using maximum likelihood, evolutionary distance, and maximum parsimony methods. Mol Biol Evol. 2011;28(10):2731-9.

35. Rawlings ND, Barrett AJ, Thomas PD, Huang X, Bateman A, Finn RD. The MEROPS database of proteolytic enzymes, their substrates and inhibitors in 2017 and a comparison with peptidases in the PANTHER database. Nucleic Acids Res. 2018;46(D1):D624-32. https://doi.org/10.1093/nar/gkx1134.

36. de Bie T, Cristianini N, Demuth JP, Hahn MW. CAFE: a computational tool for the study of gene family evolution. Bioinformatics. 2006;22(10):1269-71. https://doi.org/10.1093/bioinformatics/btl097.

37. Morgan A, Babu D, Reiz B, Whittal R, Suh LYK, Siraki AG. Caution for the routine use of phenol red - it is more than just a pH indicator. Chem Biol Interact. 2019;310:108739. https://doi.org/10.1016/j.cbi.2019.108739.
38. Ortuño-Olea L, Durán-Vargas S. The L-asparagine operon of rhizobium etli contains a gene encoding an atypical asparaginase. FEMS Microbiol Lett. 2000;189(2):177-82. https://doi.org/10.1111/j.1574-6968.2000.tb09226.x.

39. Fan $\mathrm{H}, \mathrm{Wu} \mathrm{Q}$, Wei F, Yang F, Ng BL, Hu Y. Chromosome-level genome assembly for giant panda provides novel insights into Carnivora chromosome evolution. Genome Biol. 2019;20(1):267. https://doi.org/10.11 86/s13059-019-1889-7.

40. Miga KH, Koren S, Rhie A, Vollger MR, Gershman A, Bzikadze A, et al. Telomere-to-telomere assembly of a complete human X chromosome. Nature. 2020;585(7823):79-84. https://doi.org/10.1038/s41586-020-2547-7.

41. Wang M, Zhang L, Wang Z. Chromosomal-level reference Genome of the Neotropical tree Jacaranda mimosifolia D. Don. Genome Bio Evol. 2021; 13(6):evab094. https://doi.org/10.1093/gbe/evab094.

42. Salazar AN, Gorter de Vries AR, van den Broek M, Brouwers N, de la Torre Cortes $\mathrm{P}$, et al. Chromosome level assembly and comparative genome analysis confirm lager-brewing yeasts originated from a single hybridization. BMC Genomics. 2019;20(1):916. https://doi.org/10.1186/s12 864-019-6263-3.

43. Waterhouse RM, Seppey M, Simão FA, Manni M, loannidis P, Klioutchnikov $\mathrm{G}$, et al. BUSCO applications from quality assessments to gene prediction and phylogenomics. Mol Biol Evol. 2018;35(3):543-8. https://doi.org/10.1093/ molbev/msx319.

44. Veeckman E, Ruttink T, Vandepoele K. Are we there yet? Reliably estimating the completeness of plant genome sequences. Plant Cell. 2016;28(8):175968. https://doi.org/10.1105/tpc.16.00349.

45. Dunn MJ, Kinney GM, Washington PM, Berman J, Anderson MZ. Functional diversification accompanies gene family expansion of MED2 homologs in Candida albicans. PLoS Genet. 2018;14(4):e1007326. https://doi.org/10.1371/ journal.pgen.1007326.

46. Demuth JP, Hahn MW. The life and death of gene families. BioEssays. 2009; 31(1):29-39. https://doi.org/10.1002/bies.080085.

47. Dunlop PC, Meyer GM, Ban D, Roon RJ. Characterization of two forms of asparaginase in Saccharomyces cerevisiae. J Biol Chem. 1978;253(4):1297-304. https://doi.org/10.1016/S0021-9258(17)38144-9.

48. Mahajan RV, Saran S, Saxena RK, Srivastava AK. A rapid, efficient and sensitive plate assay for detection and screening of $\mathrm{L}$-asparaginaseproducing microorganisms. FEMS Microbiol Lett. 2013;341(2):122-6. https:// doi.org/10.1111/1574-6968.12100.

49. Ghasemi Y, Ebrahiminezhad A, Rasoul-Amini S, Zarrini G, Ghoshoon MB, Raee MJ, et al. An optimized medium for screening of L-asparaginase production by Escherichia coli. Am J Biochem Biotechnol. 2008;4(4):422-4. https://doi.org/10.3844/AJBBSP.2008.422.424.

50. Chung D, Baek K, Bae SS, Jung J. Identification and characterization of a marine-derived chitinolytic fungus, Acremonium sp. YS2-2. J Microbiol. 2019; 57(5):372-80. https://doi.org/10.1007/s12275-019-8469-0.

51. Altschul SF, Gish W, Miller W, Myers EW, Lipman DJ. Basic local alignment search tool. J Mol Biol. 1990;215(3):403-10. https://doi.org/10.1016/S0022-2 836(05)80360-2.

52. Bolger AM, Lohse M, Usadel B. Trimmomatic: a flexible trimmer for Illumina sequence data. Bioinformatics. 2014;30(15):2114-20. https://doi.org/10.1093/ bioinformatics/btu170.

53. Oxford Nanopore Technologies. https://community.nanoporetech.com.

54. PoreChop. https://github.com/rrwick/Porechop.

55. Walker B, Abeel T, Shea T, Priest M, Abouellel A, Sakthikumar S, et al. Pilon: an integrated tool for comprehensive microbial variant detection and genome assembly improvement. PLoS One. 2014;9(11):e112963. https://doi. org/10.1371/journal.pone.0112963.

56. Gurevich A, Saveliev V, Vyahhi N, Tesler G. QUAST: quality assessment tool for genome assemblies. Bioinformatics. 2013;29(8):1072-5. https://doi.org/1 0.1093/bioinformatics/btt086.

57. Benson G. Tandem repeats finder: a program to analyze DNA sequences. Nucleic Acids Res. 1999;27(2):573-80. https://doi.org/10.1093/nar/27.2.573.

58. Pérez-Wohlfeil E, Diaz-del-Pino S, Trelles O. Ultra-fast genome comparison for large-scale genomic experiments. Sci Rep. 2019;9(1):1-10. https://doi. org/10.1038/s41598-019-46773-w.

59. Li H, Durbin R. Fast and accurate short read alignment with burrowswheeler transform. Bioinformatics. 2009;25(14):1754-60. https://doi.org/10.1 093/bioinformatics/btp324.

60. Quinlan AR, Hall IM. BEDTools: a flexible suite of utilities for comparing genomic features. Bioinformatics. 2010;26(6):841-2. https://doi.org/10.1093/ bioinformatics/btq033. 
61. Mach-aigner AR, Martzy R. Trichoderma reesei. Methods protocols. 1st ed; 2021. Springer Protocols

62. Frith MC. A new repeat-masking method enables specific detection of homologous sequences. Nucleic Acids Res. 2011;39(4):e23. https://doi.org/1 0.1093/nar/gkq1212.

63. Kim D, Langmead B, Salzberg SL. HISAT: a fast spliced aligner with low memory requirements. Nat Methods. 2015;12(4):357-60. https://doi.org/10.1 038/nmeth.3317.

64. Haas BJ, Papanicolaou A, Yassour M, Grabherr M, Blood PD, Bowden J, et al. De novo transcript sequence reconstruction from RNA-seq using the trinity platform for reference generation and analysis. Nat Protoc. 2013;8(8):1494512. https://doi.org/10.1038/nprot.2013.084.

65. Haas BJ, Delcher AL, Mount SM, Wortman JR, Smith RKJr, Hannick LI, et al. Improving the Arabidopsis genome annotation using maximal transcript alignment assemblies. Nucleic Acids Res. 2003;31(19):5654-66. https://doi. org/10.1093/nar/gkg770.

66. Buchfink B, Xie C, Huson DH. Fast and sensitive protein alignment using DIAMOND. Nat Methods. 2015;12(1):59-60. https://doi.org/10.1038/nmeth.3176.

67. Slater GSC, Birney E. Automated generation of heuristics for biological sequence comparison. BMC Bioinformatics. 2005;6(1):31. https://doi.org/1 0.1186/1471-2105-6-31.

68. Borodovsky M, Lomsadze A. Eukaryotic gene prediction using GeneMark hmm-E and GeneMark-ES. Curr Protoc Bioinformatics. 2011; Chapter 4(Unit-4. 6):1-10. https://doi.org/10.1002/0471250953.bi0406s35.

69. Stanke M, Keller O, Gunduz I, Hayes A, Waack S, Morgenstern B. AUGUSTUS: $a b$ initio prediction of alternative transcripts. Nucleic Acids Res. 2006;34(2): W435-W9. https://doi.org/10.1093/nar/gkl200.

70. Majoros WH, Pertea M, Salzberg SL. TigrScan and GlimmerHMM: two open source ab initio eukaryotic gene-finders. Bioinformatics. 2004;20(16):2878-9. https://doi.org/10.1093/bioinformatics/bth315.

71. Zaharia M, Bolosky WJ, Curtis K, Fox A, Patterson D, Shenker S, et al. Faster and more accurate sequence alignment with SNAP. ArXiv preprint arXiv. 2011;1111:5572 https://arxiv.org/abs/1111.5572

72. Testa AC, Hane JK, Ellwood SR, Oliver RP. CodingQuarry: highly accurate hidden Markov model gene prediction in fungal genomes using RNA-seq transcripts. BMC Genomics. 2015;16(1):170. https://doi.org/10.1186/s12864-01 5-1344-4.

73. Haas BJ, Salzberg SL, Zhu W, Pertea M, Allen JE, Orvis J, et al. Automated eukaryotic gene structure annotation using EVidenceModeler and the program to assemble spliced alignments. Genome Biol. 2008;9(1):R7. https:// doi.org/10.1186/gb-2008-9-1-r7.

74. Chan PP, Lowe TM. tRNAscan-SE: searching for tRNA genes in genomic sequences. Methods Mol Biol. 1962;2019:1-14. https://doi.org/10.1007/ 978-1-4939-9173-0_1.

75. Käll L, Krogh A, Sonnhammer ELL. A combined transmembrane topology and signal peptide prediction method. J Mol Biol. 2004;338(5):1027-36. https://doi.org/10.1016/j.jmb.2004.03.016.

76. Huerta-Cepas J, Forslund K, Coelho LP, Szklarczyk D, Jensen LJ, von Meringet $C$, et al. Fast genome-wide functional annotation through orthology assignment by eggNOG-mapper. Mol Biol Evol. 2017;34(8):211522. https://doi.org/10.1093/molbev/msx148.

77. Jones P, Binns D, Chang HY, Fraser M, Li W, McAnulla C, et al. InterProScan 5: genome-scale protein function classification. Bioinformatics. 2014;30(9): 1236-40. https://doi.org/10.1093/bioinformatics/btu031.

78. Johnson LS, Eddy SR, Portugaly E. Hidden Markov model speed heuristic and iterative HMM search procedure. BMC Bioinformatics. 2010;11(1):431. https://doi.org/10.1186/1471-2105-11-431.

79. Finn RD, Bateman A, Clements J, Coggill P, Eberhardt RY, Eddy SR, et al. Pfam: the protein families database. Nucleic Acids Res. 2014;42(D1):D222D30. https://doi.org/10.1093/nar/gkt1223.

80. Yu Y, Ouyang $Y$, Yao W. shinyCircos: an R/shiny application for interactive creation of Circos plot. Bioinformatics. 2018;34(7):1229-31. https://doi.org/1 0.1093/bioinformatics/btx763.

81. Li L, Stoeckert CJ, Roos DS. OrthoMCL: identification of ortholog groups for eukaryotic genomes. Genome Res. 2003;13(9):2178-89. https://doi.org/10.11 01/gr.1224503.

82. Edgar RC. MUSCLE: a multiple sequence alignment method with reduced time and space complexity. BMC Bioinformatics. 2004;5(1):113. https://doi. org/10.1186/1471-2105-5-113.

83. Capella-Gutiérrez S, Silla-Martínez JM, Gabaldón T. trimAl: a tool for automated alignment trimming in large-scale phylogenetic analyses.
Bioinformatics. 2009;25(15):1972-3. https://doi.org/10.1093/bioinformatics/ btp348.

84. Kumar S, Stecher G, Suleski M, Hedges SB. TimeTree: a resource for timelines, timetrees, and divergence times. Mol Biol Evol. 2017;34(7):1812-9. https://doi.org/10.1093/molbev/msx116.

85. Patro R, Duggal G, Love ML, Irizarry RA, Kingsford C. Salmon provides fast and bias-aware quantification of transcript expression. Nat Methods. 2017; 14(4):417-9. https://doi.org/10.1038/nmeth.4197.

86. Love ML, Huber W, Anders S. Moderated estimation of fold change and dispersion for RNA-seq data with DESeq2. Genome Biol. 2014;15(12):550. https://doi.org/10.1186/s13059-014-0550-8.

87. Team RC. R: a language and environment for statistical computing. 2012. http://www.R-project.org/.

\section{Publisher's Note}

Springer Nature remains neutral with regard to jurisdictional claims in published maps and institutional affiliations.

\section{Ready to submit your research? Choose BMC and benefit from:}

- fast, convenient online submission

- thorough peer review by experienced researchers in your field

- rapid publication on acceptance

- support for research data, including large and complex data types

- gold Open Access which fosters wider collaboration and increased citations

- maximum visibility for your research: over $100 \mathrm{M}$ website views per year

At BMC, research is always in progress.

Learn more biomedcentral.com/submissions 